\title{
The Universe of Chaos and Quanta
}

\author{
B.G. Sidharth \\ Centre for Applicable Mathematics \& Computer Sciences \\ B.M. Birla Science Centre, Adarsh Nagar, Hyderabad - 500063 (India) \\ Email:birlasc@hd1.vsnl.net.in; birlard@ap.nic.in
}

\begin{abstract}
In recent papers it was shown that stochastic processes in the universe as a whole lead to discrete space time at Compton scales as also non- relativistic Quantum Mechanics. In this paper, we deduce the Dirac equation and thence a unified formulation of quarks and leptons. In the process several puzzling empirical results and coincidences are shown to be a consequence of the theory. These include the discreteness of the charge, handedness of quarks, their fractional charge, confinement and masses and the handedness of neutrinos, the so called accidental relation that the classical Kerr-Newman metric describes, the field of an electron including the purely quantum mechanical gyromagnetic ratio $g=2$, as also the many large number coincidences made famous by Dirac and Weinberg's mysterious empirical formula that relates the pion mass to the Hubble Constant. A cosmology based on fluctuations related to the above stochastic spacetime discretization, consistent with latest observations is also seen to follow.
\end{abstract}

\section{Introduction}

In previous communications [1, 2, 3] an attempt was made to give a stochastic formulation as the underpinning to physical laws. The starting point was the well known Brownian random walk relation

$$
R=l \sqrt{N}
$$


which is true for the universe as a whole, $R$ being its radius, $N$ being the number of elementary particles, typically pions in the universe and $l$ the pion Compton wavelength. It was argued that (1) would be the starting point as a rationale for quantum mechanics itself. We will now carry the argument one step further and show that, on this basis we can deduce the two basic equations of quantum mechanics, namely the Dirac and Schrodinger equations and thence deduce a model for all the fundamental particles namely the quarks and the leptons. This model will bring out all peculiar characteristics of these basic particles, including features like fractional charges, handedness, confinement and so on. Hitherto these features have been purely empirical without a theoretical rationale. At the same time, it will be pointed out that in the same vein one could get a cosmological description, consistent with data and observation.

\section{An Underpinning of Quantum Theory}

We first observe that, using the well known fact that $R=c T$, where $T$ is the age of the universe, the following relation can be obtained from (1):

$$
T=\sqrt{N} \tau
$$

where $\tau=l / c$ is the pion Compton time.

Furthermore, as there are $N$ particles typically pions in the universe, it should follow that

$$
M=N m
$$

where $M$ is the mass of the universe and $m$ the pion mass. This is indeed so. We next observe that equations (1) and (2) indicate that the Compton scale is a fundamental unit of space time. Infact it was shown [2] that this quantized space time could be considered to be more fundamental than Planck's quantized energy - the latter can be shown to follow from the former. It may be mentioned that the concept of discrete space time has a long history and has been studied by several authors for example Snyder, Schild, Kardyshevsky, Bombelli, Wolfe and several others [4]- [8] As T.D. Lee observes 9], the space-time continuum is an approximation. Infact as pointed out [2, 10] such a quantized space time description, following from (11) and (2) provides a rationale for a maximal velocity and Special Relativity itself. Snyder[四] 
pointed out that discrete space time is compatible with Special Relativity and went on to show

$$
\begin{aligned}
& {[x, y]=\left(\imath a^{2} / \hbar\right) L_{z,}[t, x]=\left(\imath a^{2} / \hbar c\right) M_{x},} \\
& {[y, z]=\left(\imath a^{2} / \hbar\right) L_{x},[t, y]=\left(\imath a^{2} / \hbar c\right) M_{y},} \\
& {[z, x]=\left(\imath a^{2} / \hbar\right) L_{y},[t, z]=\left(\imath a^{2} / \hbar c\right) M_{z},}
\end{aligned}
$$

where $a$ is the minimum natural unit and $L_{x}, M_{x}$ etc. have their usual significance.

Similarly it was also deduced that

$$
\begin{gathered}
{\left[x, p_{x}\right]=\imath \hbar\left[1+(a / \hbar)^{2} p_{x}^{2}\right] ;} \\
{\left[t, p_{t}\right]=\imath \hbar\left[1-(a / \hbar c)^{2} p_{t}^{2}\right] ;} \\
{\left[x, p_{y}\right]=\left[y, p_{x}\right]=\imath \hbar(a / \hbar)^{2} p_{x} p_{y} ;} \\
{\left[x, p_{t}\right]=c^{2}\left[p_{x}, t\right]=\imath \hbar(a / \hbar)^{2} p_{x} p_{t} ; \text { etc. }}
\end{gathered}
$$

where $p^{\mu}$ denotes the four momentum.

While equations (4) bring out the non commutative nature of space time geometry, equations (5) show a departure from the usual quantum mechanical commutation relations. However as $a \rightarrow 0$, we recover the usual theory, as expected.

Using now the fact that the minimum natural unit $a$ is the Compton wavelength, $l=\hbar / m c$, and the fact that at these extreme scales $|\vec{p}|=m c$, it follows from (5)

$$
\left[x, p_{x}\right]=2 \imath \hbar
$$

and similar equations.

The right hand side of (6) is double the usual value - as if the spin has been halved or the coordinate doubled. This is the typical double connectivity of spin half.

This is suggestive of the surprising fact that Quantum Mechanical spin would arise from quantized space time. This can be confirmed as follows: We consider a linear transformation of the wave function,

$$
\left|\psi^{\prime}>=U(R)\right| \psi>
$$


If the transformation $R$ is a simple infinitesimal coordinate shift in Minkowski space we will get, from (7), as is well known[11, 12],

$$
\psi^{\prime}\left(x_{j}\right)=\left[1+\imath \epsilon\left(\imath \epsilon_{l j k} x_{k} \frac{\partial}{\partial x_{j}}\right)+0\left(\epsilon^{2}\right)\right] \psi\left(x_{j}\right)
$$

We next consider the commutation relations (田), taking $a$ to be the Compton wavelength. We can easily verify that the choice

$$
t=\left(\begin{array}{ll}
1 & 0 \\
0 & -1
\end{array}\right), \vec{x}=\left(\begin{array}{cc}
0 & \vec{\sigma} \\
\vec{\sigma} & 0
\end{array}\right)
$$

provides a representation for the coordinates $x$ and $t$ apart from any scalar factors. We can immediately recognize that equation (9) is a representation of the Dirac $\gamma$ matrices. Substitution of (9) in (8) now leads to the Dirac equation,

$$
\left(\gamma^{\mu} p_{\mu}-m c^{2}\right) \psi=0
$$

because

$$
E \psi=\frac{1}{\epsilon}\left\{\psi^{\prime}\left(x_{j}\right)-\psi\left(x_{j}\right)\right\}, E=m c^{2},
$$

where $\epsilon=\tau$ (cf.ref. $[\mathbb{8}]$ ). Thus we have shown that given minimum space time intervals of the order of the Compton wavelength and time, a simple coordinate transformation leads to the Dirac equation. (Alternatively, we could have considered the operator $\exp \left(-\imath \epsilon^{\mu} p_{\mu}\right)$.)

It must be mentioned that the usual derivation of the Dirac equation itself is heuristic, in the sense that the square root operator is not very transparent, while at the same time the emergence of a four component spinor instead of a two component spinor and the negative energy solutions and non Hermitian position operators are puzzling, as also the standard interpretation that on averaging over Compton scales, these Zitterbewegung effects and non hermitian operators disappear. All this now becomes transparent - physics begins outside these minimum quantized space time Compton scales so that we obtain a physical interpretation only after averaging over these intervals and we encounter unphysical effects at smaller scales [13, 14, 15, 9]). That is, a space time continuum is an approximation that breaks down within the Compton scales.

It is ofcourse well known that in the non relativistic limit $v / c<<1$, the Dirac equation goes over to the Schrodinger equation. Infact it was shown that the 
above Compton scale cut off[2] explains the otherwise adhoc and therefore unsatisfactory features of Nelson's stochastic derivation of the Schrodinger equation [16.

All this is a reconfirmation of the fact pointed out earlier that space time quantization at the Compton scale provides an underpinning for quantum mechanics.

\section{Quarks and Leptons}

At the Compton scale, as is known, the negative energy two spinor $\chi$ of the full four rowed Dirac spinor $\left(\theta_{\chi}\right)$, (where $\theta$ denotes the positive energy two spinor), begins to dominate. Further under reflections, while $\theta$ goes to $\theta, \chi$ behaves like a psuedo-spinor[12],

$$
\chi \rightarrow-\chi
$$

Hence the operator $\frac{\partial}{\partial x^{\mu}}$ acting on $\chi$, a density of weight $N=1$, has the following behaviour 13,

$$
\frac{\partial \chi}{\partial x^{\mu}} \rightarrow \frac{1}{\hbar}\left[\hbar \frac{\partial}{\partial x^{\mu}}-N A^{\mu}\right] \chi
$$

where,

$$
A^{\mu}=\hbar \Gamma_{\sigma}^{\mu \sigma}=\hbar \frac{\partial}{\partial x^{\mu}} \log (\sqrt{|g|})
$$

$\Gamma^{\prime}$ 's being the usual Christoffel symbols.

We can easily identify $N A^{\mu}$ in (11) with the electromagnetic four potential. (That $A^{\mu}$ is a four gradient poses no problem 13]. The fact that $N=1$ explains why the charge is discrete). We can also immediately see the emergence of the metric tensor and the resulting potential.

On the face of it (12) is mathematically identical to Weyl's Unification formulation of electromagnetism and gravitation [17]. However there is a very important difference - (11) and (12) arise from the purely quantum mechanical behaviour of the Dirac spinor (cf. also 18]) of the equation (10).

We now use the fact that the metric tensor $g_{\mu \nu}$ resulting from (12) satisfies an inhomogenous Poisson equation 17, 19,

$$
\nabla^{2} g_{\mu \nu}=G \rho u_{\mu} u_{\nu}
$$


It immediately follows that

$$
g_{\mu \nu}=G \int \frac{\rho u_{\mu} u_{\nu}}{\left|\vec{r}-\vec{r}^{\prime}\right|} d^{3} \vec{r}
$$

where now we require the volume of integration to be the Compton volume. Alternatively we observe that as is known[20, 21], for the Dirac equation (10) we can get the Hamilton-Jacobi formulation and thence equation (13) when the energy term as in (11) and (12) is introduced in (10).

In any case (14) can be recognized as the linearized general relativity equation [19]. From (14) we can get the usual gravitational potential for $r \equiv|\vec{r}-\vec{r}|>>$ the Compton wavelength,

$$
g_{00}=-\frac{G m}{r}
$$

As shown elsewhere [13, 14, 18, 22], given the linearized equation of General Relativity, (14) was the starting point of a geometrized formulation of Fermions leading to the Kerr-Newman metric and which explains the remarkable and supposedly coincidental fact that the Kerr-Newman metric describes the field of an electron including the anomalous gyro magnetic ratio $g=2$. In this connection two points need to be noted. Firstly an electron is indeed a Kerr-Newman Black Hole except for a naked singularity. Secondly in the derivation of the Kerr-Newman metric, an imaginary shift of origin is used which as Newman confessed is as yet inexplicable 23. Both these have an immediate parallel in the Zitterbewegung and non Hermitian position operators of the Quantum Mechanical electron. Once the minimum Compton scale as above is recognized, these inexplicable features disappear.

All this was also shown to lead to a unified description of electromagnetism, gravitation and strong interactions 18, 22.

We now show how a unified description of quarks and leptons can be obtained from (14).

From (12) and (14) we get

$$
A_{0}=G \hbar \int \frac{\partial}{\partial t} \frac{\left(\rho u_{\mu} u_{\nu}\right)}{\left|\vec{r}-\vec{r}^{\prime}\right|} d^{3} r \approx \frac{e e^{\prime}}{r}
$$

for $\left|\vec{r}-\vec{r}^{\prime}\right|>>$ the Compton wavelength where $e^{\prime}=e$ is the test charge.

Further, from (16), as in the discrete case, $d \rho u_{\mu} u_{\nu}=\Delta \rho c^{2}=m c^{2}$ and $d t=\hbar / m c^{2}$, we get

$$
A_{0}=\frac{e^{2}}{r} \sim G \frac{\hbar}{r} \frac{\left(m c^{2}\right)^{2}}{\hbar}
$$


or

$$
\frac{e^{2}}{G m^{2}} \sim 10^{40}
$$

(17) is the well known but hitherto purely empirical relation expressing the ratio of the gravitational and electromagnetic strengths. Here we have deduced (17) from theory.

It was pointed out[18] that starting from (14) one could obtain the KerrNewman metric which describes the field of the electron, and via the equations $(11)$, (12), (14), (15), (16) and (17) we have affected the unified description of electromagnetism and gravitation.

If in (14) we consider distances of the order of the Compton wavelength, it was shown that we will get instead of (15), a QCD type potential

$$
\begin{array}{r}
4 \quad \eta^{\mu v} \int \frac{T_{\mu \nu}\left(t, \vec{x}^{\prime}\right)}{\left|\vec{x}-\vec{x}^{\prime}\right|} d^{3} x^{\prime}+(\text { terms independent of } \vec{x}), \\
+2 \quad \eta^{\mu v} \int \frac{d^{2}}{d t^{2}} T_{\mu \nu}\left(t, \vec{x}^{\prime}\right) \cdot\left|\vec{x}-\vec{x}^{\prime}\right| d^{3} x^{\prime}+0\left(\left|\vec{x}-\vec{x}^{\prime}\right|^{2}\right) \propto-\frac{\propto}{r}+\beta r
\end{array}
$$

where $T_{\mu \nu} \equiv \rho u_{\mu} u_{\nu}$. Equation (18) can lead to a reconciliation of electromagnetism and strong interactions 22]. For this we need to obtain a formulation for quarks from the above considerations. This is what we will do now.

We first use the fact that the doubleconnectivity or spin half of the electron leads naturally to three dimensional space 19, 24, that is at asymptotic distances far outside the Compton wavelength. However this doubleconnectivity or spinorial behaviour breaks down at Compton scales and we need to consider low dimensionality namely two and one dimensions. Using the well known fact that each of the $T_{i j}$ in (16) is given by $\frac{1}{3} \epsilon$ [25], $\epsilon$ being the energy density, it follows immediately from (16) that the charge would be $\frac{2}{3} e$ or $\frac{1}{3} e$ in two or one dimensions, exactly as for quarks. At the same time as we are now at the Compton scale, automatically these fractionally charged particles are confined. This indeed is expressed by the confining part of the QCD potential (18). Further, at the Compton scale, as noted earlier 12 we encounter predominantly the negative energy components of the Dirac spinor with, opposite parity so that these quarks would show neutrino type handedness, which indeed is true.

Thus at one stroke, all the peculiar empirical characteristics of the quarks for which as Salam had noted[26], there was no theoretical rationale, can now 
be deduced from theory. We can even get the correct order of magnitude estimate for the quark masses. For this we rewrite (18) by multiplying the expression by $\frac{1}{m}$ (owing to the factor $\frac{\hbar^{2}}{2 m}$ ), and at the same time go over to units $c=\hbar=1$. This will facilitate comparison with standard literature[27]. (18) then becomes

$$
\frac{4}{m} \quad \eta^{\mu v} \int \frac{T_{\mu v}}{r} d^{3} x+2 m \quad \eta^{\mu v} \int T_{\mu v} r d^{3} x \equiv-\frac{\propto}{r}+\beta r
$$

where now, $\alpha \sim O$ (团) and $\beta \sim O\left(m^{2}\right)$, where $m$ is the mass of the quark in agreement with standard literature.

We now observe that at the Compton scale, for the fractionally charged quarks $e^{2}$ in (16) goes over to $\frac{e^{2}}{9} \sim 10^{-3}$ in the units being considered. So (16) can be written as

$$
\frac{1}{r} \approx 10^{3} G \hbar \int \frac{\partial}{\partial t} \frac{\rho u_{\mu} u_{\nu}}{\left|\vec{r}-\vec{r}^{\prime}\right|} d^{3} r
$$

Comparing (20) for the quarks with the Coulumb term of the QCD potential (19) and the electrostatic potential for the electron given in (16), we can immediately see that the quark mass would be $\sim 10^{3} \times$ the electron mass, which ofcourse is in order.

The above description is equally valid for neutrinos if we remember that they have vanishingly small mass. So their Compton wavelength is very large and by the same argument as above, we encounter predominantly the negative energy components of the Dirac spinor which have opposite parity, that is the neutrinos display handedness.

This brings us to the question of weak interactions. So far we have dealt with interactions which in the conventional language, are mediated by mass less intermediary Bosons.

We will now argue that a weak electromagnetic interaction with a massive intermediary $m_{w} \sim 100 m_{p}$, where $m_{p}$ is the proton mass, is entirely consistent with the foregoing considerations.

In the early days of the electro weak theory 28] it was already realized that this was indeed the case for the weak coupling constant roughly set equal to the electromagnetic coupling constant:

$$
G_{w} \equiv g^{2} / m_{w}^{2} \approx \frac{10^{-5}}{m_{p}^{2}} g m^{-2}
$$


where $G_{w}$ is the Fermi local weak coupling constant.

(21) can be deduced from (17), where it should now be borne in mind that

(17) is not an adhoc relation, but rather a deduction from the theory. Infact with $g^{2} \approx 10^{-1}$, we get from (17) and (21) $G_{w} \approx 10^{43}$ as required. We will come back to this point again in section 5 .

\section{Cosmological Considerations}

We now come to the field of Cosmology and will consider the fluctuational creation of particles, which will be seen to be closely linked with the stochastic minimum space time intervals. Our starting point is the fact that as is well known there is a background Zero Point Field (ZPF), which as noted by Vigier and others indeed has been considered to be what drives fluctuations 29, 30, 31]. It is also known that the energy of the fluctuations of for example the magnetic field in a region of length $l$ is given by 19

$$
B^{2} \sim \frac{\hbar c}{l^{4}}
$$

where $\vec{B}$ is the magnetic field strength.

Taking now $l$ to be the Compton wavelength, for a volume $\sim l^{3}$, the energy of the resultant particle is given by

$$
\text { Energy } \sim \frac{\hbar c}{l}=m c^{2},
$$

which ofcourse is correct. In other words the entire energy of an elementary particle of mass $m$ is generated by the fluctuations alone 14. We now show that all this leads to a perfectly consistent cosmology.

As in the usual theory we equate the Newtonian gravitational potential energy (which is dominant on a large scale) of the pion in a three dimensional isotropic sphere of pions of radius $R$, the radius of the universe, with the rest energy of the pion, to get the well known relation

$$
R=\frac{G M}{c^{2}}
$$

where $M$ can be obtained from (3).

We now use the fact that the fluctuation in the particle number is of the order $\sqrt{N}$ 14, 32, 33, while a typical time interval of uncertainity is $\sim \hbar / m c^{2}$ 
as seen above. (That is particles induce more particles by fluctuations).

It must be mentioned that this is somewhat in the spirit of the work of Prigogine and his coworkers 34, 35]: Heisenberg's Uncertainity gives rise to production of energy over short intervals of time, leading to a one way creation of particles. In the words of Prigogine [36], "...in this perspective, time is eternal... has neither a beginning nor an end. We come therefore to a position which unifies elements contained in the two traditional uses of cosmology: The Steady State Theory and the standard Big Bang approach."

In this light we combine the usual fluctuation in particle number with the minimum uncertainity in time to consider the creation of particles, from the background ZPF.

This leads to the relation [14,

$$
\dot{N} \equiv \frac{d N}{d t}=\sqrt{N} \frac{m c^{2}}{\hbar}
$$

whence an integration between $t=0$ and $t=T$, the present age of the universe, gives,

$$
T=\frac{\hbar}{m c^{2}} \sqrt{N}
$$

which is just equation (2).

We have rederived equation (2) but from a slightly different point of view. Earlier we had started with the random walk equation (11) and then using the relation $R=c T$, we got equation (2). In the case of (23) we started with the well known fact that the fluctuation in particle number is $\sim \sqrt{N}$. Infact as $N$ itself can be arbitrary in principle, this shows that the two apparently different formulations are the same. This will be further confirmed below.

We now observe that from (22) we get,

$$
\dot{R}=\frac{G \dot{N} m}{c^{2}}+\frac{N m}{c^{2}} \dot{G}
$$

As will be seen (cf. equation (30) $),|\dot{G}| \sim N^{-1}$ while $\dot{N}$ from above is $\sim \sqrt{N}$ and the second term is half the first and with opposite sign. So

$$
\frac{d R}{d t} \approx H R
$$

and, from (24),

$$
\frac{d^{2} R}{d t^{2}} \approx H^{2} R+\dot{H} R=\lambda R, \lambda \approx 0
$$


where $H$ in (24) can be identified with the Hubble Constant, and as can be seen from the above (equations (22) ff.),

$$
H=\frac{G m^{3} c}{2 \hbar^{2}}
$$

Both the terms in (25) are equal and opposite and cancel out, that is $\lambda$ vanishes.

Comparing (25) with the well known equation of General Relativistic Cosmology 25], we can see that $\lambda$ plays the role of the cosmological constant which vanishes. However, in view of the order of magnitude calculations, strictly speaking,

$$
\lambda \leq 0\left(H^{2}\right)
$$

Indeed (27) agrees with observation 19.

We emphasize that equations (22) and (23) show that in this formulation, the correct radius and age of the universe can be deduced given $N$ as the sole cosmological or large scale parameter.

Further, equation (26) or equivalently,

$$
m \approx\left(\frac{\hbar^{2} H}{G c}\right)^{1 / 3}
$$

which is considered to be a mysterious and as yet unexplained coincidence is rather a consequence. We will comment on this shortly.

To proceed we observe that the fluctuation of $\sim \sqrt{N}$ (due to the ZPF) leads to an extra electrostatic energy $\frac{e^{2} \sqrt{N}}{R}$ which is balanced by the energy of the electron itself viz., $m_{e} c^{2}[32]$. Whence we get:

$$
R=\sqrt{N} l
$$

and

$$
N_{1}=\sqrt{N}=\frac{e^{2}}{G m^{2}} \approx 10^{40}
$$

which are just (11) and (17).

We have thus rederived the random walk equation (11) also, using fluctuation in particle number, and in the process rederived (17) also.

If we combine $(28)$ and $(22)$, we get,

$$
\frac{G m}{l c^{2}}=\frac{1}{\sqrt{N}}
$$


So, as noted in the derivation of (24), $|\dot{G}| \sim N^{-1}$. If we combine (30) and (23), we get Dirac's equation 14]

$$
G \alpha T^{-1}
$$

It must be mentioned that, as argued by Dirac and Melnikov[37] we treat $G$ as the variable, rather than the quantities $m$ (orl), cand $\hbar$ (the micro physical constants), because of their central role in atomic (and sub atomic) physics. Further, using (30) in (29), with $N_{1}=\sqrt{N}$, we can see that the charge $e$ also is independant of time or $N$. So $e$ also must be added to the list of microphysical constants.

Next if we use $G$ from (30) in (26), we can see that

$$
H=\frac{c}{l} \quad \frac{1}{\sqrt{N}}
$$

Thus apart from the fact that $H$ has the same inverse time dependance on $T$ as $G$, (32) shows that given the microphysical constants, and $N$, we can deduce the Hubble Constant also.

Using (2) and (28), we can deduce that

$$
\rho \approx \frac{m}{l^{3}} \quad \frac{1}{\sqrt{N}} \propto T^{-1}
$$

Thus we have the scenario of an ever expanding (and possibly accelerating) universe 38 with, as can be seen from (33), decreasing density. Latest observations of distant supernovae by different teams of observers confirm all this 39, 40.

It should also be noted that in the above considerations we have deduced theoretically the so called Dirac large number of coincidences, which have been supposed to be accidental features. However the resulting cosmology of Dirac was, as is well known 25] inconsistent owing to the fact that there were relations like, $R \propto T^{1 / 3}$, which ofcourse is not correct. But then it must be remembered that Dirac had considered a fixed number of particles.

\section{Discussion}

1. It was already pointed out in section 2 that if the minimum cut off tends to zero, we recover the conventional picture. In particular, we can recover 
the usual Big Bang formulation in the limit $\tau \rightarrow 0$. Indeed in this case we can see from the equation leading to (23) that $\frac{d N}{d t} \rightarrow \infty$, indicating a singular creation. More accurately, we take the Planck scale $\left(l_{P}, \tau_{P}\right)$ instead of the pion scale $(l, \tau)$ as above as the zero point scale as in quantum gravity [41. The Planck density $\rho_{P}$ is given by another mysterious "coincidence",

$$
\rho_{P} \times l^{3}=M
$$

It would also follow that the number of Planck masses in the above volume $l^{3}$ is $N^{\prime} \sim 10^{60}$ but then we have to consider the fact that in the physical time period $\tau$ corresponding to the length $l$, there are $\frac{\tau}{\tau_{P}} \sim 10^{20}$ Planck life times, so that the number of particles in the physical interval $(l, \tau)$ is $N \sim 10^{80}$, which is the total particle number. In the above derivation, we consider the semi classical Quantum Gravity picture in conjunction with the Quantum Mechanical Compton scale.

Thus we recover the entire mass and also the entire number of particles in the universe, with a singular creation, as in the Big Bang theory. However in this limit we cannot deduce the large number coincidences or the Weinberg formula (26).

2. We have seen the intimate relationship between the Brownian type relations (1) and (2), as also the fluctuation in particle number $\sim \sqrt{N}$ on the one hand and purely quantum mechanical micro physics as also cosmological considerations on the other. This is typified by not just equations (1) and (2), but also equations like (26), (29) and (30). This is an expression of what has been called the micro-macro nexus 14 or stochastic holism, in contrast to the conventional idea of a whole built out of parts as for example could be typified by an equation like (3).

3. One may wonder how the cosmic microwave background radiation comes in. Infact it has been shown that the observed cosmic microwave background radiation is consistent in the above scheme 42, 14, 43.

4. An important question that arises is that of the observed mass spectrum. It has been shown, in a phenomenological scheme consistent with decay modes that such a mass spectrum can indeed be consistently obtained 44 in the light of foregoing considerations.

5. It is interesting to note that given the random walk relation (1), using the relation (23) (rather than (2)), we can deduce that $R=c T$. In other words the velocity of light itself is seen to emerge from the above stochastic 
formulation. Indeed this is not surprising because as noted in section 2, such a maximum velocity is a consequence of discrete space time.

6. In section 3 we obtained equation (13) (or (14)) which was the linearized equation of General Relativity. As is known starting from such a linearized theory we could bootstrap our way to the full equation of General Relativity 19, 45].

7. In effect the minimum space time intervals have fudged the singularities of General Relativity - for the Kerr-Newman formulation as also for the Big Bang. Indeed as has been observed, these singularities of General Relativity pose the greatest challenge of modern Physics. Such singularities are now seen to be a result of going down to arbitrarily small space time intervals, a process which is not legitimate even in Quantum Mechanics. At the same time, the ultra violet divergences of Quantum Theory also disappear, which indeed was one of the original motivations for space-time discretization (cf.refs. [甘], 9]).

8. We could derive the weak interactions from a slightly different point of view. If these are mediated by an intermediate particle of mass $m_{w}$ and Compton wavelength $l_{w}$ then, as in the run up to equation (28) and (29) using the fluctuation in particle number we can deduce instead of (29), now the equation[28]

$$
g^{2} \sqrt{N_{\nu}} l_{w}^{2} \approx m_{\nu} c^{2}
$$

where $N_{\nu}$ is the number of neutrinos and $m_{\nu}$ is the neutrino mass.

We use the fact that 46,32

$$
m_{\nu} \approx 10^{-8} m_{e}
$$

that is the mass of the neutrino is about one hundred millionth that of the electron, as indeed has been confirmed by the recent super Kamiokande experiments. It is also known that $N_{\nu} \sim 10^{90}$ so that from (35) we get,

$$
g^{2} l_{w}^{2} \sim 10^{-59}
$$

which infact gives back equation (21) for the weak interaction.

\section{Conclusion}

Using the Brownian theory we have deduced minimum space time intervals which have directly lead to the Dirac equation and a unified formulation of 
not just quantum theory but quarks and leptons. In the process hitherto purely empirical results like the discreteness of the charge, the handedness of the neutrino, or the handedness of quarks, their fractional charge, confinement and masses have been deduced from the theory. The so called accidental relations like the classical Kerr-Newman metric providing a description of the field of the electron including the purely quantum mechanical gyromagnetic ratio $g=2$ as also the various large number coincidences and Weinberg's mysterious formula are also now seen to be natural consequences of the theory.

This apart a cosmology consisting with observation is also seen to follow.

\section{References}

[1] Sidharth, B.G., "Stochastic Conservation Laws" to appear in Chaos, Solitons and Fractals.

[2] Sidharth, B.G., "The Chaotic Universe" to appear in Chaos, Solitons and Fractals.

[3] Sidharth, B.G., "Quantized Space Time and Time's Arrow", to appear in Chaos, Solitons and Fractals.

[4] Snyder, H.S., Phys. Rev. $\underline{71}$ (1), 1947, p.38ff.

[5] Schild, A., Discrete space-time and integral Lorentz transformations, Physical Review 73, 1947, p.414-415.

[6] Bombelli, L., Lee, J., Meyer, D., and Sorkin, R.D., Phys.Rev.Lett. 59, 521, 1987.

[7] Kadyshevskii, V.G., Soviet Physics Doklady 7 (12), 1963, p.1138ff.

[8] Wolf, C., Nuovo.Cim., 109B (3), 1994, p.213ff.

[9] Lee, T.D., Phys Lett. 12 2B, 1983, 217.

[10] Hakim, R., J.Math.Phys. 9, 1968, p.1805ff.

[11] Schweber, S.S., "An Introduction to Relativistic Quantum Field Theory", Harper and Fow, New York, 1961. 
[12] Bjorken, J.D., and Drell, S.D., (1964) "Relativistic Quantum Mechanics", McGraw Hill, New York.

[13] Sidharth, B.G., Ind.J. Pure \& Appd.Phys., $\underline{35}$ (7), 1997, 456-471.

[14] Sidharth, B.G., Int.J. of Mod.Phys.A 13(15), 1998, pp2599ff.

[15] Wolf, C., Hadronic Journal, 15, 1992, pp321ff.

[16] Nelson, E., Phys.Rev. 150, 1966, 1079ff.

[17] Bergmann, P.G., "Introduction to the Theory of Relativity", PrenticeHall (New Delhi), 1969, p248ff.

[18] Sidharth, B.G., Gravitation \& Cosmology, 4 (2) (14), 1998, $158 \mathrm{ff}$.

[19] Misner, C.W., Thorne, K.S., and Wheeler, J.A., Gravitation, Freeman (San Francisco), 1973.

[20] Koga, T., Int.J.Th.Phys, Vol.13, No.4 1975, pp.271-278.

[21] Koga, T., Int.J.Th.Phys, Vol.12, No.3 1975, pp.205-215.

[22] Sidharth, B.G., To appear in Mod.Phys.Lett.A.

[23] Newman, E.T., J.Math. Phys. 14 (1), 1973, p102.

[24] Penrose, R., "Angular Momentum: An approach to combinational space-time" in, "Quantum Theory and Beyond", Ed., Bastin, T., Cambridge University Press, Cambridge, 1971, pp151ff.

[25] Narlikar, J.V., "Introduction to Cosmology", Foundation Books, New Delhi, 1993, p.57.

[26] Salam, A., "Unification of Fundamental Forces", Cambridge University Press, Cambridge, 1990.

[27] Lee, T.D., "Particle Physics and Introduction to Field Theory", Harwood Academic Publishers, New York, 1981.

[28] Bernstein, J., "Elementary Particles and their currents", Freeman, San Francisco, 1968. 
[29] Vigier, J.P., Lett Nuovo Cim 29 (14), 1980, pp467ff.

[30] De Pena, L., "Stochastic Processes applied to Physics...", Ed., B Gomex, World Scientific, Singapore, 1983.

[31] Gaeta, G., Phys.Lett.A. 175, 267-268, 1993.

[32] Hayakawa, S., Suppl of PTP Commemmorative Issue, 1965, 532-541.

[33] Huang, K., "Statistical Mechanics", Wiley Eastern, New Delhi, 1975.

[34] Prigogine, I., and Gunzig, E., Nature $\underline{330}, 1987,621$.

[35] Gunzig, E., et al, Annalen der Physik 115, 1978, 78.

[36] Prigogine, I., End of Uncertainity (Freepress, New York 1997).

[37] Melnikov, V.N., International Journal of Theoretical Physics, $\underline{33}$ (7), 1994, 1569-1579.

[38] Sidharth, B.G., International Journal of Theoretical Physics, 37 (4), 1998, 1307-1312.

[39] Perlmutter, S., et.al., Nature $\underline{391}$, 1998, 51-54.

[40] Coles, Peter and Ellis George, F.R., "Is the Universe Open or Closed?", Cambridge University Press, Cambridge, 1997.

[41] Padmanabhan, T., Classical and Quantum Gravity, 4LL, 1987, 107.

[42] Sidharth, B.G., in "Frontiers of Quantum Physics", Eds., Lim, S.C., et al, Springer Verlag, Singapore, 1998.

[43] Narlikar, J.V., Did the Universe Originate in a Big Bang? in "Cosmic Perspectives", Eds., Biswas, S.K., Mallik, D.C.V., and Vishveshwara, C.V., Cambridge University Press, Cambridge, 1989, p.109ff.

[44] Sidharth, B.G., and Lobanov, Yu, Frontiers of Fundamental Physics (Universities Press, Hyderabad 1998).

[45] Sidharth, B.G., Non Linear World, 4 , 1997, 157-161. 
[46] Sidharth, B.G., "Neutrino Mass and an Ever Expanding Universe (An Irreverent Perspective)", "Workshop on Neutrino Physics", University of Hyderabad, November 1998 (In Proceedings). 